\title{
Acute coronary syndrome in patients undergoing anticancer therapies: A single-center, controlled case study
}

\author{
Izabela Nabiałek-Trojanowska, ${ }^{1,2, B-D}$, Alicja Dąbrowska-Kugacka ${ }^{2, E}$, Zuzanna Lewicka-Potocka, ${ }^{1,2,-E}$, \\ Yasmina Abdulaziz ${ }^{2, B-D}$, Anna Szerszyńska ${ }^{2, B-D}$, Grzegorz Raczak²,E, Ewa Lewicka ${ }^{2, A, E, F}$ \\ ${ }^{1}$ First Department of Cardiology, Medical University of Gdańsk, Poland \\ ${ }^{2}$ Department of Cardiology and Electrotherapy, Medical University of Gdańsk, Poland \\ A - research concept and design; $\mathrm{B}$ - collection and/or assembly of data; $\mathrm{C}$ - data analysis and interpretation; \\ $\mathrm{D}$ - writing the article; $\mathrm{E}$ - critical revision of the article; $\mathrm{F}$ - final approval of the article
}

Address for correspondence

Ewa Lewicka

E-mail: elew@gumed.edu.pl

Funding sources

None declared

Conflict of interest

None declared

Received on January 1, 2019

Reviewed on April 20, 2019

Accepted on June 27, 2019

Published online on December 18, 2019

Cite as

Nabiałek-Trojanowska I, Dąbrowska-Kugacka A, Lewicka-Potocka Z. Acute coronary syndrome in patients undergoing anticancer therapies: A single-center, controlled case study. Adv Clin Exp Med. 2019;28(12):1667-1673. doi:10.17219/acem/110316

DOI

10.17219/acem/110316

Copyright

Copyright by Author(s)

This is an article distributed under the terms of the

Creative Commons Attribution 3.0 Unported (CC BY 3.0)

(https://creativecommons.org/licenses/by/3.0/)

\begin{abstract}
Background. Anticancer therapies can be accompanied by cardiovascular complications, including acute coronary syndrome (ACS). In turn, the presence of cancer can influence therapeutic decisions if ACS occurs.

Objectives. The aim of the study was to analyze ACS treatment in patients with cancer.

Material and methods. The study consisted of a retrospective analysis based on the medical records of patients who were admitted due to ACS, with cancer diagnoses. Patients currently undergoing cancer treatment or having treatment which ended up to 6 months before the ACS were included. They were compared to a control group consisting of consecutive patients admitted for ACS during the same period, but who did not have a diagnosis of cancer; they were matched with the experimental group in terms of age, gender and clinical type of ACS.
\end{abstract}

Results. Thirty-two consecutive cancer patients ( $70 \pm 9$ years; $53 \%$ men) met the inclusion criteria. In 22 of them (69\%), ACS occurred during their cancer treatment, and in $10(31 \%)$, it presented within 6 months of completing cancer treatment. Upon hospital admission, 19 (59\%) cancer patients complained of dyspnea and 7 of typical angina, while in the control group 28, (87\%) and 4 (13\%) reported such symptoms, respectively.The clinical manifestation of ACS was NSTEMI in 16 patients (50\%), UA in 10 (31\%) and STEMI in 5 (15.6\%). Coronary angiography was done in 25 (78\%) of the cancer patients and in all members of the control group. Percutaneous coronary angioplasty (PCA) was performed in 17 (53\%) and 23 (72\%) of the patients from the respective groups. The median time to percutaneous coronary intervention (PCI) was $10 \mathrm{~h}$ ( 30 min-10 days) among the cancer patients and $7.5 \mathrm{~h}$ among the control group (30 min-6 days). There were no PCl-related complications or severe bleeding in both groups. In-hospital mortality was $6.25 \%$ in the cancer group and there were no reported hospital deaths in the control group.

Conclusions. Dyspnea is the most common symptom of ACS in cancer patients who are treated invasively too rarely: the presence of cancer and active anticancer treatment should not limit the management of ACS in accordance with current guidelines.

Key words: acute coronary syndrome, anticancer therapy, STEMI, NSTEMI, cancer disease 


\section{Introduction}

Anticancer therapies can be accompanied by cardiovascular complications, including acute coronary syndrome (ACS). A recently published registry has shown that cancer survivors, compared to the general population, are at a higher risk of cardiovascular morbidity and mortality and that they represent a large group of patients undergoing percutaneous coronary intervention (PCI): 1 in every 13 patients. ${ }^{1}$ Cancer at various stages and treated with various anticancer therapies is reported in about $15 \%$ of patients with ACS. ${ }^{2}$

The association between cancer and ACS is complex and multifactorial. Many cancers have risk factors in common with coronary artery disease: older age, male sex, smoking and obesity. ${ }^{3}$ Cancer itself leads to a prothrombotic state, oxidative stress and the progression of atherosclerosis. ${ }^{4}$ Additionally, anticancer treatment may increase thrombotic risk and lead to cardiotoxic effects, since chemotherapy and radiotherapy exert pro-inflammatory and vasospastic effects. ${ }^{5,6}$

Optimal ACS treatment in cancer patients can be difficult, as these patients are at risk of both stent thrombosis and the bleeding that is often increased due to thrombocytopenia. In clinical practice, cancer reported in anamnesis can change the treatment plan due to the unknown prognosis of life length and the higher risk of bleeding, as well as to thrombotic events which may accompany the treatment.

The aim of our study was to analyze the treatment of ACS administered to patients with cancer during or soon after the end of their anticancer therapy.

\section{Methods}

Based on the hospital database, 3 investigators independently reviewed the discharge cards, medical history reports and all available medical documents of patients hospitalized in our Cardiology Departments because of ACS from January 2012 to December 2017. The keywords for the search were ACS, ST-elevation myocardial infarction (STEMI), non-ST-segment elevation myocardial infarction (NSTEMI), unstable angina (UA), cancer, and neoplasm, using ICD codes C0-C97, D37-D48 and I20-I22.

Next, we searched for patients in whom ACS presented during or soon after (up to 6 months after the end of anticancer therapy or treatment). The medical records of these patients were carefully screened to analyze the ACS treatment. The control group consisted of consecutive patients admitted for ACS during the same period, but without a diagnosis of cancer. They were matched with the study group in terms of age, gender and clinical type of ACS. The statistical analyses of continuous data were performed using a t-test in the case of normal distributions, and non-parametric tests (Mann-Whitney U test) in the case of non-normally distributed or ordinal data. P-values $<0.05$ were considered statistically significant.

\section{Results}

Overall, 8,327 records with ACS reported were retrieved from the hospital database, of which 441 records were of patients with a diagnosis of cancer. Finally, 32 records based on the inclusion criteria and deemed adequate for the purpose of our study were screened in detail. The control group consisted of 32 patients with a similar age and sex distribution and the same frequency of ACS types.

We analyzed the data obtained from 32 consecutive cancer patients at a mean age of $70 \pm 9$ years (58-88 years) 17 of whom (53.1\%) were men admitted from the emergency department due to ACS occurring a median of 7 months after their cancer diagnosis. In the cancer group, the most common disease was lung cancer, diagnosed in 9 patients (28.1\%). From the remaining patients, breast cancer was in $6(18.8 \%)$, prostate cancer in $4(12.5 \%)$, colon cancer in 4 (12.5\%), gastric cancer in 2 , ovarian cancer in 2 , head and neck cancer in 2, endometrial cancer in 1, non-Hodgkin lymphoma in 1, and chronic myeloid leukemia in 1. For 29 of the patients, it was a newly diagnosed tumor, while in the remaining 3 it was a recurrence of cancer. In 22 patients (69\%), ACS occurred during anticancer treatment: chemotherapy in 15 patients, hormonotherapy in 4, combined radiation and chemotherapy in 2 , and during immunotherapy in 1 . In 10 patients (31\%), ACS developed within 6 months of the end of anticancer treatment: in 5 patients who had previously undergone chemotherapy and after thorax radiation in the other 3 patients. Twenty-two patients $(68.7 \%)$ had received chemotherapy, and the most commonly used anticancer drugs which could potentially affect coronary arteries or lead to arterial thrombosis were platinum compounds and fluoropyrimidines, administered in $31.2 \%$ and $18.6 \%$ of patients, respectively. Data on the chemotherapy agents which were administered is shown in Table 1. Thirteen patients (40.6\%) had recently undergone radiotherapy. Of the 13, 7 (21.9\%) had undergone thorax irradiation and in 2 patients the cancer recurred more than 10 years after radiotherapy. Seven patients $(21.9 \%)$ received combined chemotherapy and radiotherapy.

Almost all cancer patients (30 (94\%)) presented with at least 1 cardiovascular risk factor or comorbidity. Coronary artery disease was reported in 13 (40\%) patients, and previous myocardial infarction in 3 patients. In the control group, all patients had at least 1 cardiovascular risk factor or comorbidity, and coronary artery disease was diagnosed in $14(44 \%)$ patients, while previous myocardial infarction was found in 11 patients. The clinical characteristics of both groups are shown in Table 2 .

Upon admission to the hospital, 19 patients (59\%) from the control group presented with dyspnea, and 7 (22\%) with typical angina. In the control group, the main ACS symptom was typical angina in 28 patients (87\%), while dyspnea was reported by 4 patients (13\%). The most common clinical ACS manifestation was NSTEMI, diagnosed 
Table 1. Anticancer therapy in the study group of cancer patients

\begin{tabular}{|l|c|}
\multicolumn{1}{|c|}{ Chemotherapy agents } & Number of patients \\
\hline Platinum compounds & 5 \\
cisplatin & 4 \\
carboplatin & 1 \\
oxaliplatin & \\
\hline Fluoropyrimidines & 2 \\
5-fluorouracil & 3 \\
capecitabine & 1 \\
gemcitabine & \\
Anthracyclines & 3 \\
doxorubicin & 2 \\
\hline Alkylating agents & \\
cyclophosphamide & 2 \\
Antimicrotubulale agents & 1 \\
docetaxel & \\
paclitaxel & 1 \\
\hline VEGF inhibitors & 1 \\
bevacizumab & \\
Vinca alkaloids & \\
vincristine & \\
vinorelbine & \\
\hline Hydroxycarbamide & \\
\hline
\end{tabular}

VEGF - vascular endothelial growth factor.

Table 2. Clinical characteristics of patients with acute coronary syndrome and cancer (study group) and those without cancer (control group)

\begin{tabular}{|c|c|c|c|}
\hline Parameter & $\begin{array}{l}\text { Cancer group } \\
\qquad(\mathrm{n}=32)\end{array}$ & $\begin{array}{l}\text { Control group } \\
\quad(n=32)\end{array}$ & $\mathrm{p}$-value \\
\hline Males, n (\%) & $17(53)$ & $17(53)$ & $p=1.0$ \\
\hline Age [years] & $70 \pm 9$ & $70 \pm 9$ & $p=1.0$ \\
\hline $\begin{array}{l}\text { Previous coronary } \\
\text { artery disease, n (\%) }\end{array}$ & $13(40)$ & $14(44)$ & $p=1.0$ \\
\hline $\begin{array}{l}\text { Arterial hypertension, } \\
\mathrm{n}(\%)\end{array}$ & $23(72)$ & $30(94)$ & $p=0.1$ \\
\hline Dyslipidemia, n (\%) & $12(37.5)$ & $30(94)$ & $p<0.001$ \\
\hline Diabetes, n (\%) & $10(32)$ & $18(56)$ & $p=0.08$ \\
\hline Smoking history, n (\%) & $15(54)$ & $10(31)$ & $p=0.14$ \\
\hline $\begin{array}{l}\text { Obesity } \\
\left(\mathrm{BMI}>30 \mathrm{~kg} / \mathrm{m}^{2}\right), \mathrm{n}(\%)\end{array}$ & $6(19)$ & $9(28)$ & $p=0.7$ \\
\hline STEMI, n (\%) & $5(15.6)$ & $5(15.6)$ & $p=1.0$ \\
\hline NSTEMI, n (\%) & $10(31.3)$ & $10(31.3)$ & $p=1.0$ \\
\hline Unstable angina, n (\%) & $16(50)$ & $16(50)$ & $p=1.0$ \\
\hline $\begin{array}{l}\text { Type } 2 \text { myocardial } \\
\text { infarction, } \mathrm{n}(\%)\end{array}$ & $1(3.1)$ & $1(3.1)$ & $p=1.0$ \\
\hline
\end{tabular}

BMI - body mass index; STEMI - ST-elevation myocardial infarction; NSTEMI - non-ST-segment-elevation myocardial infarction.

in 16 cancer patients (50\%). Unstable angina (UA) had occurred in 10 patients (31.3\%) and STEMI in 5 (15.6\%). In 1 patient with severe anemia, a type 2 myocardial infarction (MI) had occurred. The incidence of the various clinical ACS types was the same as in the control group.

In the cancer group, coronary angiography was done in 25 patients (78\%). Seventeen patients (53\%) underwent percutaneous coronary angioplasty (PCA), with the implantation of a drug-eluting stent (DES) in $12 \mathrm{pa}$ tients and a bare metal stent (BMS) in the remaining 5 patients. None of the patients were treated with balloon angioplasty (POBA) or referred for coronary artery bypass grafting (CABG). In 6 patients, a third-generation DES was implanted. In the control group, all patients were referred for coronary angiography. Twenty-three (72\%) were treated with PCA: DES implantation in 21 patients and BMS implantation in 1 , while 1 patient was treated with POBA. In 4 patients, a third-generation DES was implanted. As in the cancer group, none of the patients were referred for CABG. In the cancer group, PCA with stent implantation was performed in 3 patients (60\%) with STEMI, 9 with NSTEMI and 5 with UA, while in the control group it was in 5 (100\%), 12 and 6 patients, respectively.

The median time from hospital admission to PCA among the cancer patients was $10 \mathrm{~h}$; it was $105 \mathrm{~min}$ in the patients with STEMI (ranging from $30 \mathrm{~min}$ to $10 \mathrm{~h}$ ), $14.5 \mathrm{~h}$ in patients with NSTEMI (from 30 min to 10 days) and $13.5 \mathrm{~h}$ in those with UA (from $30 \mathrm{~min}$ to 4 days). In the control group, the median time between admission and PCA was $7.5 \mathrm{~h}$ ( $\mathrm{p}=0.6 \mathrm{vs}$ the cancer group); it was $35 \mathrm{~min}$ in the patients with STEMI (ranging from $30 \mathrm{~min}$ to $8 \mathrm{~h} ; \mathrm{p}=0.6$ ), $11.5 \mathrm{~h}$ in the patients with NSTEMI (from 30 min to 6 days; $\mathrm{p}=0.8$ ) and $5 \mathrm{~h}$ in those with UA (from $60 \mathrm{~min}$ to $42 \mathrm{~h} ; \mathrm{p}=0.1$ ).

Coronary catheterization was performed using the radial approach in all but 1 cancer patient, in whom femoral access was used. There were no PCA-related complications or serious bleeding. In 2 patients, bleeding in the arterial puncture area was noted (1 patient with femoral access), which was self-limiting and did not require medical intervention. In the control group, coronary angiography was performed using the radial approach in 26 patients (81\%), via a brachial artery in 2 and via a femoral artery in 4 (12\%). No PCA-related complications or bleeding were reported in the control group. Data on the ACS treatment administered to individual patients are shown in Table 3.

Among the cancer patients, aspirin was administered to 29 (94\%), clopidogrel to $23(74 \%)$ and 22 (71\%) patients obtained double antiplatelet therapy (DAPT). Nine patients did not receive antiplatelet treatment because they reported anemia or severe bleeding in anamnesis, and 1 was allergic to aspirin. Enoxaparine was administered to 16 patients (51.6\%). A loading dose of $300 \mathrm{mg}$ of aspirin was administered to 20 patients and $600 \mathrm{mg}$ of clopidogrel to 23. Ticagrelor and prasugrel were not used in our patients. Triple antithrombotic therapy, involving aspirin, clopidogrel and enoxaparine, was used in 13 patients. None of the patients received a vitamin $\mathrm{K}$ antagonist (VKA) or a novel oral anticoagulant (NOAC). In 1 patient, data about antiplatelet and antithrombotic treatment was not obtained. Gastrointestinal bleeding occurred in 1 patient and nasal bleeding in another 1 , but medical intervention or prolongation of hospitalization were not required. Statin 
Table 3. Hematological data and treatment strategy in patients with acute coronary syndrome (ACS) and cancer (study group) and in those without cancer (control group)

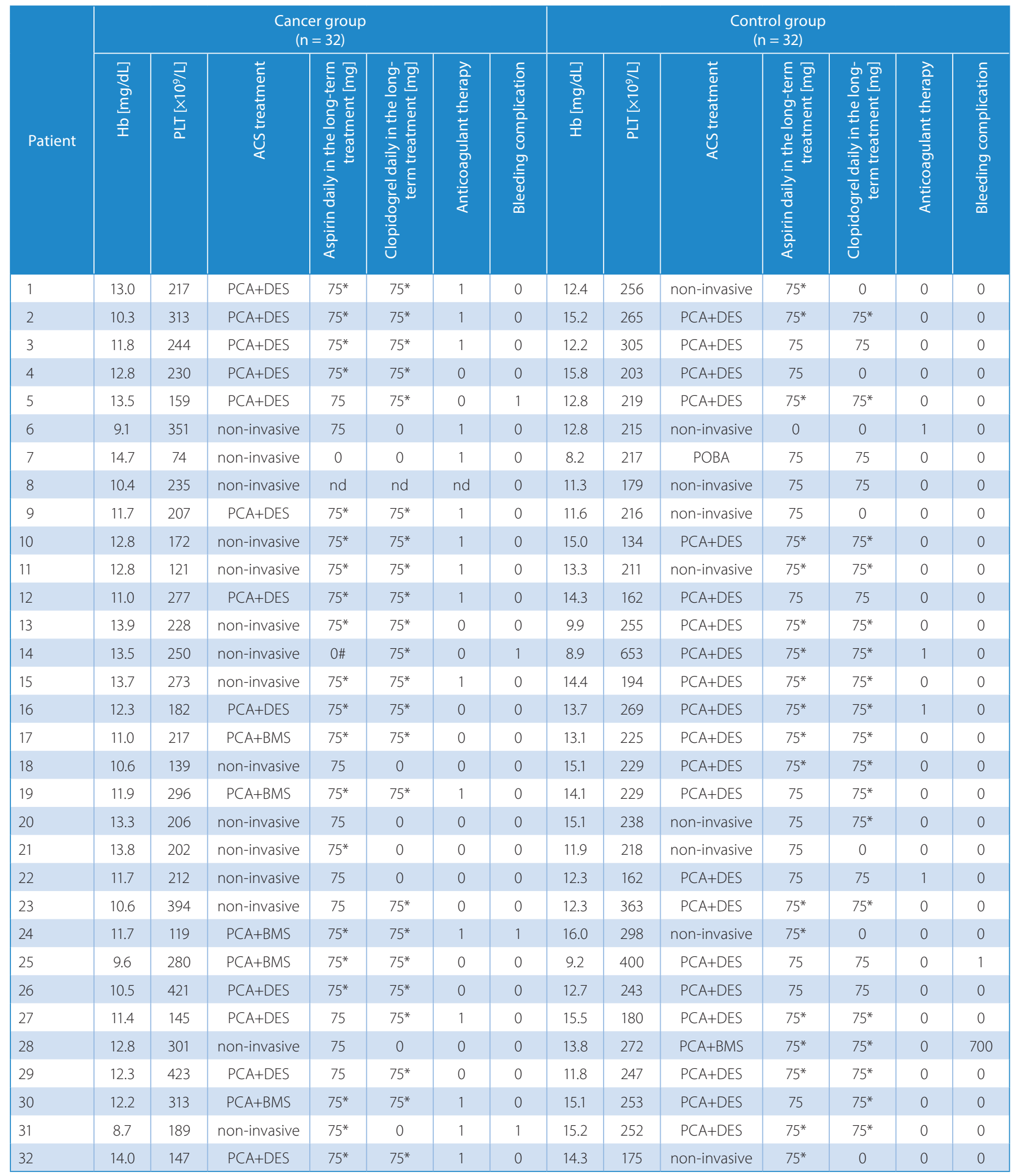

$\mathrm{Hb}$ - hemoglobin concentration; PLT - platelet count; PCA - percutaneous coronary angioplasty; DES - drug-eluting stent; BMS - bare metal stent; nd - no data; 1 - 'Yes'; 0 -'No'; *application of a loading dose of 300 mg of aspirin and/or 600 mg of clopidogrel; \# - aspirin allergy.

was administered to 25 patients $(80.6 \%), \beta$-blockers in 28 (87.1\%) and an angiotensin converting enzyme inhibitor (ACEI) or angiotensin receptor blocker (ARB) was administered to 23 (74.2\%).
In the control group, 31 (97\%) patients received aspirin, 25 (78\%) clopidogrel, 1 (3\%) received ticagrelor, and 26 (81\%) were given DAPT. A loading dose of $300 \mathrm{mg}$ of aspirin was administered to 18 patients, and $600 \mathrm{mg}$ of clopidogrel 
to another 18 patients. One patient with type 2 myocardial infarction due to tachyarrhythmia was treated conservatively and received only anticoagulant therapy (enoxaparine). In 3 other patients, antithrombotic therapy was administered: warfarine in 2 patients and dabigatran in 1 . One patient had nasal bleeding which was treated conservatively, with no significant drop in hemoglobin concentration. Thirty patients (94\%) received statin, 25 (78\%) $\beta$-blockers and 29 (91\%) patients were administered ACEI or ARB.

None of the patients from either study group suffered from severe thrombocytopenia, and hemoglobin concentration in the 2 groups varied from $8.7 \mathrm{mg} / \mathrm{dL}$ to $14 \mathrm{mg} / \mathrm{dL}$ and from $8.2 \mathrm{mg} / \mathrm{dL}$ to $16.0 \mathrm{mg} / \mathrm{dL}$, respectively. In echocardiographic examination performed before hospital discharge, the mean left ventricular ejection fraction was $55 \pm 12 \%(33-66 \%)$ in the cancer group and $50 \pm 10 \%$ $(25-74 \%)$ in the control group $(\mathrm{p}=0.16)$.

In-hospital mortality among all patients admitted to our cardiology departments due to ACS in the study period was $6.16 \%$. In the cancer group, during a median of 5 days (3-31 days) of hospitalization 2 patients died (6.25\%) due to sudden cardiac arrest and pulseless electrical activity. There were no more deaths from ACS during the following 30 days, though data regarding outcome were not collected on 2 patients. In the control group, the median duration of hospitalization was 6 days (2-22 days) and there were no hospital deaths or deaths from ACS during the following 30 days.

\section{Discussion}

The results of the study indicate that the majority of patients with ACS presenting during or soon after anticancer treatment can be treated according to the current ACS guidelines, without adversely affecting in-hospital prognosis or the duration of hospitalization. The vast majority of cancer patients received antiplatelet therapy, and even if DAPT or enoxaparine was used, it was not accompanied by an increased risk of bleeding during hospitalization. Most patients were given a $\beta$-blocker, ACEI or ARB and statin. However, coronary angiography was performed too rarely (in only $78 \%$ of the cancer patients) and only $53 \%$ underwent PCA with stent implantation.

The pathogenesis of ACS among cancer patients includes the impact of classic cardiovascular risk factors, as well as the influence of a prothrombotic state, oxidative stress and tumor-induced atherosclerosis. ${ }^{4}$ The effects of anticancer treatment (i.e., chemotherapy, radiotherapy or surgery) must also be considered. The majority of our cancer patients presented with cardiovascular risk factors or comorbidities, with no significant differences in comparison with the controls (apart from dyslipidemia) which was more frequent among the control group. In addition, the median time from cancer diagnosis was 7 months, which may affect the occurrence of ACS. It has been reported that the incidence of ACS in patients with newly diagnosed cancer increases in the first 6 months from diagnosis and then decreases after a year to increase again in more advanced cancer stages. ${ }^{6,7}$ In our patients, we must also consider the influence of recent anticancer therapy on the development of their ACS, as chemotherapy and radiotherapy can exert prothrombotic, pro-inflammatory and vasospastic effects. ${ }^{5,6,8}$ We focused on an early cardiac manifestation of anticancer therapy complication, although 2 patients from our group presented with tumor recurrence years after thorax radiotherapy. In this case, the mechanism of ACS may differ, and it could be a result of fibrosis or calcification within the coronary arteries.

Many chemotherapeutic agents predispose one to ACS, as they may provoke coronary vasospasm, endothelial damage or arterial thrombosis and they may aggravate atherosclerosis. ${ }^{9}$ A number of chemotherapeutic agents may lead to acute coronary events, mainly cisplatin, 5-fluorouracil, vincristine, rituximab, and BCR-ABL-directed tyrosine kinase inhibitors. Likewise, paclitaxel, capecitabine, VEGF inhibitors, erlotinib, nilotinib, and ponatinib have been reported to exert vascular toxicity, especially in coronary arteries. The time of ischemia onset varies widely. It may occur within hours of infusion or several days afterwards. ${ }^{10}$ Cisplatin-related risk can persist even after the end of chemotherapy. ${ }^{11}$

Modern radiotherapy aims to focus the radiation beam on the invaded tissue; complications of such treatment are rarer than with the previously used methods. The mechanism of radiation-related damage to the coronary arteries is similar to chemotherapy, and it could be an effect of endothelial injury, coronary vasospasm, atherosclerotic plaque rupture or thrombosis. ${ }^{9,12}$ Such injuries are usually located in the ostia and proximal segments of the coronary arteries. ${ }^{13}$ The risk of radiation-related coronary artery disease depends on the radiation dosage and the volume of the irradiated heart. ${ }^{9}$ It can manifest early, during or soon after the end of radiation, or with a delay, even after $10-15$ years.

As opposed to patients from the control group, who reported angina as the main ACS symptom, many of the cancer patients presented with dyspnea at hospital admission, which is consistent with the observations of other authors. ${ }^{14-16}$ Radio- and chemotherapy-related neurotoxicity can affect the ability to feel pain, so in effect patients after anticancer treatment complain of angina less often. The occurrence of ACS manifests as dyspnea in 44.3\% of cancer patients, chest pain in $30.3 \%$ and hypotension in $22.7 \% .{ }^{16}$ As a result of either the higher prevalence of silent ischemia or the altered perception of angina after anticancer treatment, cancer patients seek emergency care after some delay. Emphasis should be placed on cardiac check-up before anticancer treatment with any cardiotoxicity potential and regularly after the end of the treatment in order to reveal complications of anticancer therapy at an early stage. 
The most common clinical manifestation of ACS in our patients was NSTEMI. This finding is in accordance with other studies, which reported that in $85 \%$ of cancer patients the ACS manifested as NSTEMI and in 15\% as STEMI. ${ }^{17}$ Conservative treatment of ACS in cancer patients leads to poor survival rate. ${ }^{18}$ An analysis of treatment in patients with metastatic cancer who developed STEMI or NSTEMI revealed that invasive treatment with PCI resulted in a 2- to 3 -fold reduction in in-hospital mortality. ${ }^{19}$ However, the results of one study indicated that cancer patients undergoing PCI due to STEMI had poorer survival after 1 year (10.7\% vs $5.4 \%$ ) and higher cardiac mortality, which especially pertained to those diagnosed up to 6 months before the onset of ACS. ${ }^{20}$ The optimal treatment in this group remains indefinite, because there is no data available on cancer patients in the PCI registries. Formerly, cancer patients were excluded from most major randomized, controlled ACS trials. According to the available data, PCI offers a better prognosis in this group of patients, but the need for antiplatelet therapy after stenting should be taken into account in treatment planning. Double antiplatelet therapy can cause hemorrhagic complications and anemia, especially gastrointestinal and urinary bleeding.

It has been proven that early PCI improves outcomes in ACS independently from the patients' group. ${ }^{21}$ In the general population, the frequency of PCI has increased during the last 2 decades, from $11.9 \%$ to $60.8 \%$ of patients admitted with STEMI. This corresponds with significantly lower 30-day mortality and overall mortality. ${ }^{22}$ In Poland, according to a recent registry, PCI was performed in $96.2 \%$ of patients with STEMI, and in $76.3 \%$ of patients with NSTEMI or UA. ${ }^{23}$ In our groups, $78 \%$ of cancer patients were referred for coronary angiography, and 53\% were treated with PCA and stent implantation, while in the control group it was $100 \%$ and $72 \%$ of patients, respectively. Our data indicates that invasive treatment of ACS is less common in patients with cancer, despite current guidelines. Moreover, in STEMI patients with cancer, the median time from admission to PCI was $105 \mathrm{~min}$, while it was $35 \mathrm{~min}$ in the control group. Guidelines recommend that the interval between arrival at the hospital and intracoronary balloon inflation (door-to-balloon time) during primary PCI should be 90 min or less. In the STEMI registry, the median door-to-balloon time was $83 \mathrm{~min}^{24}$; thus, it was too late in our patients.

A study comparing PCI outcomes in patients with and without cancer history proved that those reporting cancer in anamnesis received stents less often. Moreover, a delay in invasive treatment, assessed by the time between diagnosis and balloon inflation, was evident in the cancer group. In this study, higher early cardiac mortality was linked to anemia and cardiogenic shock during PCA, which occurred more frequently in cancer patients. ${ }^{20}$

In the majority of study patients, PCI was performed by the radial approach. One of 2 bleeding events at the puncture site in our group occurred in a cancer patient on whom PCI was performed via the femoral artery. According to the literature, femoral artery access is associated with a higher risk of bleeding, even with the use of vascular closure devices after coronary angiography. ${ }^{25}$ The femoral approach should be used in patients with abnormal Allen's test results in both hands, with arterial lines, those who have had bilateral mastectomy or multiple radial procedures and in those on hemodialysis. Radial artery access is preferred for others. ${ }^{26}$

The small number of bleeding events in our patients, which were self-terminated and clinically insignificant, may be due to the fact that none of the patients had severe thrombocytopenia during the treatment of ACS. This fact allowed aspirin to be safely administered in $94 \%$ of cancer patients and DAPT to be used in $71 \%$, similarly to the control group ( $97 \%$ and $78 \%$ of patients, respectively). There is no platelet count which limits coronary catheterization, ${ }^{26}$ and the use of aspirin in ACS treatment among cancer patients with a platelet count below $100,000 / \mu \mathrm{L}$ was associated with a higher 7 -day survival rate compared to those who did not receive aspirin (90\% vs 6\%). ${ }^{27}$ Double antiplatelet therapy with clopidogrel can be used in patients with a platelet count of 30,000-50,000/ $\mu \mathrm{L}$. Ticagrelor and prasugrel should not be used in cancer patients due to the high risk of bleeding in this group. ${ }^{17}$

In $70 \%$ of cancer patients who underwent PCA, a drugeluting stent was implanted, which is contrary to other studies reporting that BMSs are used more often in cancer patients. ${ }^{13,20}$ The antiproliferative effects of chemotherapy may delay the normal endothelization process observed among non-cancer patients after stent implantation, ${ }^{26}$ which may favor the use of DES in cancer patients with sufficient prognosis. Drug-eluting stent has lower rates of stent thrombosis ${ }^{18}$ and with third-generation DES the duration of DAPT can be shortened to 3-6 months in ACS patients. However, in cancer patients with a platelet count from $10,000 / \mu \mathrm{L}$ to $30,000 / \mu \mathrm{L}$ or if DAPT cannot be used or in those demanding surgery or chemotherapy within the next 4 weeks, balloon angioplasty should be considered. ${ }^{26} \mathrm{With}$ balloon angioplasty, DAPT is required for at least 2 weeks. $^{26}$

The vast majority of our patients were treated with a $\beta$-blocker, ACEI or ARB and statin. It was reported that not only aspirin use, but also $\beta$-blocker use, in cancer patients as in the general population, improves survival rates in ACS. ${ }^{16}$ For unknown reasons, $\beta$-blockers are less likely to be administered to cancer patients. ${ }^{20}$ Each cancer patient with ACS should be considered for optimal therapy with an antiplatelet drug or drugs, statin, ACEI or ARB and a $\beta$-blocker.

The most important limitation of our study is the small size of the cancer group and the lack of long-term observation after hospital discharge. Our data does not include the stage of cancer or planned further anticancer treatment, though this was not the focus of our study. The short 
observation period may have resulted in the low frequency of bleeding complications in the time of recommended DAPT therapy after PCI. The data was collected from our hospital database based on the medical recognition on the information cards at hospital discharge. If a diagnosis of cancer was missing on this card, the patient may not have been included in the analysis. This may to some extent explain the small number of patients from our hospital database who met the inclusion criteria for the study. As our sample is small, conclusions regarding the population of cancer patients with ACS should only be drawn with special caution. We included data on in-hospital mortality among ACS patients without cancer who were treated in our cardiology departments, but no direct comparison was made with the cancer group.

\section{Conclusions}

Our data suggests that cancer patients with ACS should be treated according to the current guidelines for ACS in the general population, taking into consideration additional factors related to cancer. Data regarding ACS management in cancer patients is still lacking, as the current information is most often based on small population studies and expert consensus. According to our results, patients with ACS onset during or shortly after anticancer therapy are too rarely treated invasively. Moreover, those with STEMI are referred for coronary angiography too late after hospital admission. The presence of cancer and active anticancer treatment should not limit the effective and safe treatment of ACS.

\section{ORCID iDs}

Izabela Nabiałek-Trojanowska (1) https://orcid.org/0000-0001-9986-2265 Alicja Dąbrowska-Kugacka (1) https://orcid.org/0000-0002-5546-7818 Zuzanna Lewicka-Potocka (1) https://orcid.org/0000-0002-7716-1190 Yasmina Abdulaziz (1) https://orcid.org/0000-0002-2659-8245 Anna Szerszyńska (iD) https://orcid.org/0000-0002-4381-865X Grzegorz Raczak (1) https://orcid.org/0000-0001-8535-8658 Ewa Lewicka (1) https://orcid.org/0000-0002-0162-2659

\section{References}

1. Landes U, Kornowski R, Bental T, et al. Long-term outcomes after percutaneous coronary interventions in cancer survivors. Coron Artery Dis. 2017:28(1):5-10.

2. Goldberg R, Chen H-Y, Saczynski J, et al. The impact of cardiac and noncardiac comorbidities on the short-term outcomes of patients hospitalized with acute myocardial infarction: A population-based perspective. Clin Epidemiol. 2013;5:439-448.

3. Hess CN, Roe MT. Treatment of coronary artery disease in cancer survivors. Coron Artery Dis. 2017;28(1):1-2.

4. Mozzini C, Garbin U, Fratta Pasini AM, Cominacini L. An exploratory look at NETosis in atherosclerosis. Intern Emerg Med. 2017;12(1):13-22.

5. Hasin T, lakobishvili Z, Weisz G. Associated risk of malignancy in patients with cardiovascular disease: Evidence and possible mechanism. Am J Med. 2017;130(7):780-785.

6. Navi BB, Reiner AS, Kamel H, et al. Risk of arterial thromboembolism in patients with cancer. J Am Coll Cardiol. 2017;70(8):926-938.

7. Yeh ETH, Chang H-M. Cancer and clot: Between a rock and a hard place. J Am Coll Cardiol. 2017;70(8):939-941.
8. Caine GJ, Stonelake PS, Lip GYH, Kehoe ST. The hypercoagulable state of malignancy: Pathogenesis and current debate. Neoplasia. 2002;4(6):465-473.

9. Zamorano JL, Lancellotti P, Rodriguez Muñoz D, et al. 2016 ESC Position Paper on cancer treatments and cardiovascular toxicity developed under the auspices of the ESC Committee for Practice Guidelines. Eur Heart J. 2016;37(36):2768-2801.

10. Barac A, Murtagh G, Carver JR, et al. Cardiovascular health of patients with cancer and cancer survivors. J Am Coll Cardiol. 2015;65(25): 2739-2746.

11. Herrmann J, Yang EH, Iliescu CA, et al. Vascular toxicities of cancer therapies: The old and the new. An evolving avenue. Circulation. 2016;133(13):1272-1289.

12. Wrona A, Dziadziuszko $R$, Jassem J, et al. Radioterapia a ryzyko powikłań ze strony układu sercowo-naczyniowego. In: Szymański F, Filipiak K, eds. Nieklasyczne Czynniki Ryzyka Chorób Układu Sercowo-Naczyniowego w Gabinecie Lekarza Praktyka. Warsaw: ITEM Publishing; 2017:137-158.

13. Lancellotti P, Nkomo VT, Badano LP, et al. Expert consensus for multi-modality imaging evaluation of cardiovascular complications of radiotherapy in adults: A report from the European Association of Cardiovascular Imaging and the American Society of Echocardiography. Eur Heart J Cardiovasc Imaging. 2013;14(8):721-740.

14. Munoz E, Iliescu G, Vejpongsa P, et al. Takotsubo stress cardiomyopathy: Good news in cancer patients? J Am Coll Cardiol. 2016;68(10): 1143-1144.

15. Roffi M, Patrono C, Collet J-P, et al. 2015 ESC Guidelines for the management of acute coronary syndromes in patients presenting without persistent ST-segment elevation. Eur Heart J. 2016;37(3):267-315.

16. Yusuf SW, Daraban N, Abbasi N, et al. Treatment and outcomes of acute coronary syndrome in the cancer population. Clin Cardiol. 2012; 35(7):443-450.

17. Banasiak W, Zymliński R, Undas A. Optimal management of cancer patients with acute coronary syndrome. Polish Arch Intern Med. 2018;128(4):244-253.

18. Iliescu C, Tsitlakidou D, Giza DE, Marmagkiolis K. Primary percutaneous coronary interventions in cancer patients. Cancer Res Front. 2017;3(1):64-71.

19. Guddati AK, Joy PS, Kumar G. Analysis of outcomes of percutaneous coronary intervention in metastatic cancer patients with acute coronary syndrome over a 10-year period. J Cancer Res Clin Oncol. 2016;142(2):471-479.

20. Velders MA, Boden $\mathrm{H}, \mathrm{Hofma} \mathrm{SH}$, et al. Outcome after ST elevation myocardial infarction in patients with cancer treated with primary percutaneous coronary intervention. Am J Cardiol. 2013;112(12):1867-1872.

21. Gale CP, Allan V, Cattle BA, et al. Trends in hospital treatments, including revascularisation, following acute myocardial infarction, 2003-2010: A multilevel and relative survival analysis for the National Institute for Cardiovascular Outcomes Research (NICOR). Heart. 2014;100(7):582-589.

22. Puymirat E, Simon T, Steg PG, et al. Association of changes in clinical characteristics and management with improvement in survival among patients with ST-elevation myocardial infarction. JAMA. 2012;308(10):998-1006.

23. Wojtkowska I, Stępińska J, Stępień-Wojno M, et al. Current patterns of antithrombotic and revascularisation therapy in patients hospitalised for acute coronary syndromes. Data from the Polish subset of the EPICOR study. Kardiol Pol. 2017;75(5):445-452.

24. Rathore SS, Curtis JP, Chen J, et al. Association of door-to-balloon time and mortality in patients admitted to hospital with ST elevation myocardial infarction: National cohort study. BMJ. 2009;338(19): b1807-b1807.

25. Sciahbasi A, Fischetti D, Picciolo A, et al. Transradial access compared with femoral puncture closure devices in percutaneous coronary procedures. Int J Cardiol. 2009;137(3):199-205.

26. Iliescu CA, Grines CL, Herrmann J, et al. SCAI Expert consensus statement: Evaluation, management, and special considerations of cardiooncology patients in the cardiac catheterization laboratory (endorsed by the Cardiological Society of India, and Sociedad Latino Americana de Cardiologia Intervencion. Catheter Cardiovasc Interv. 2016;87(5): E202-223.

27. Sarkiss MG, Yusuf SW, Warneke $C L$, et al. Impact of aspirin therapy in cancer patients with thrombocytopenia and acute coronary syndromes. Cancer. 2007;109(3):621-627. 